\title{
The influence of lapses of attention on working memory capacity
}

\author{
Nash Unsworth $^{1}$ - Matthew K. Robison ${ }^{1}$
}

Published online: 8 October 2015

(C) Psychonomic Society, Inc. 2015

\begin{abstract}
In three experiments, the influence of lapses of attention on working memory (WM) capacity measures was examined. Participants performed various change detection tasks while also reporting whether they were focused on the current task or whether they were unfocused and mind-wandering. Participants reported that they were mind-wandering roughly $27 \%$ of the time, and when participants reported mind-wandering, their performance was worse compared to when they reported being on-task. Low WM capacity individuals reported more mind-wandering and lapses of attention than high WM capacity individuals, and mind-wandering and filtering abilities were shown to make independent contributions to capacity estimates. These results provide direct support for the notion that the ability to focus attention on-task and prevent lapses of attention is an important contributor to performance on measures of WM capacity.
\end{abstract}

Keywords Working memory · Lapses of attention · Individual differences

A great deal of prior work has demonstrated that working memory (WM) can maintain roughly three to four items at a given time, and there are substantial individual differences in the number of items that can be maintained (Cowan, 2001; Cowan et al., 2005; Unsworth \& Engle, 2007). Importantly, these individual differences have been found to predict performance on a wide swath of measures ranging from low-level

Nash Unsworth nashu@uoregon.edu

1 Department of Psychology, University of Oregon, Eugene, OR 97403, USA attention tasks to higher level reasoning (Cowan et al., 2005; Unsworth et al., 2014). Recent research suggests that this covariation is due, in part, to variability in both attention control abilities and differences in capacity (Cowan, Fristoe, Elliot, Brunner, \& Saults, 2006; Unsworth et al., 2014). That is, low WM capacity individuals are poorer at actively maintaining task goals and item representations in the face of internal and external distraction compared to high WM capacity individuals (Engle \& Kane, 2004), and low WM capacity individuals cannot maintain as many representations as high WM capacity individuals (Cowan et al., 2005).

Despite initial evidence that both capacity and attention control are important, it is still unclear how attention control influences estimates of capacity. That is, during standard WM tasks, like change detection tasks, it is not clear how or why attention control would be needed. In these tasks an array of objects is briefly presented, followed by a test array, and participants are required to indicate whether the test array has changed from the initial array. When one to three objects are presented, performance tends to be quite high. However, as the number of objects increases above four, performances drastically deteriorates. Theoretically, these tasks provide fairly accurate estimates of an individual's storage capacity. What, then, is the role for attention control in these tasks? Prior work has suggested that attention control may be needed when both targets and distractors are presented, and thus attention control is needed to select targets and filter out the distractors (Vogel, McCollough, \& Machizawa, 2005). Likewise, research has suggested that attention control is needed to actively maintain items over the brief delay (C. C. Morey et al., 2011; Unsworth \& Robison, 2015). Finally, a third potential role for attention control is the need to maintain and sustain attention on task to prevent trial-to-trial fluctuations of attention. Indeed, recent modeling work has suggested that in order to model errors (and reaction times), particularly 
on small set sizes, one must incorporate a lapse parameter whereby occasionally participants experience lapses of attention and must resort to guessing (Donkin et al., 2013; R. D. Morey, 2011; Rouder et al., 2008). The notion that lapses of attention influence performance on WM measures is consistent with recent work that demonstrated that pretrial pupil diameter (a measure of alertness and inattention) is smaller on trials preceding errors than on trials preceding correct responses, even for very small set sizes (Unsworth \& Robison, 2015). Likewise fluctuations in pretrial pupil size were a significant predictor of variability in estimates of WM capacity, suggesting that lapses of attention influence performance on working memory measures, and that low WM capacity individuals experience more lapses than do high WM capacity individuals (Unsworth \& Robison, 2015).

Although the prior modeling and pupillometry work suggests the influence of lapses of attention on WM capacity, more direct evidence is needed. In particular, what is needed is a better indication of when participants are experiencing lapses of attention and how these lapses influence task performance. Typically, lapses are inferred by poor performance, such as exceptionally long reaction times (Larson \& Alderton, 1990; Unsworth et al., 2010; Weissman et al., 2006) or trials where accurate performance is much lower than normal (Adam et al., 2015). Another way of assessing lapses is to simply ask participants if they are focused on the task or if they are experiencing a lapse of attention due to mind-wandering (Smallwood \& Schooler, 2006). Specifically, prior research has used thought-probe techniques in which participants are periodically presented with thought probes during a task. Participants and are required to report whether their attention was currently focused on-task or whether they were mind-wandering. This research has consistently found that not only do participants report extensive mind-wandering during tasks, but these self-reports of attentional state are correlated with actual performance, such that self-reports of less focused attention are associated with lower levels of performance (McVay \& Kane, 2009; Smallwood \& Schooler, 2006).

Recently, Mrazek et al. (2012) found that participants report mind-wandering during complex WM span tasks (e.g., reading and operation span), and these mind-wandering self-reports were negatively related to performance such that participants who reported more mind-wandering performed more poorly on the complex span measures. These results provide initial support for the notion that mind-wandering occurs during WM tasks and that lower WM individuals mind-wander more during WM tasks. However, it is important to note that Mrazek et al. (2012) only measured mindwandering during complex span tasks and did not measure mind-wandering during other measures of WM, such as change detection tasks. Although complex span and change detection tasks can both be considered as measures of WM, they are only moderately correlated, typically load on separate factors, account for partially separate sources of variance in other cognitive constructs, and account for different aspects of WM with change detection tasks providing better measures of the number of items that can be maintained (e.g., Shipstead et al., 2014; Unsworth et al., 2014). Furthermore, given differences in the nature of the tasks it is possible that mindwandering occurs more readily in complex span tasks than in change detection tasks. That is, in complex span tasks tobe-remembered items are interspersed with some form of distracting activity (such as solving math operations). This inclusion of the distractor task may make it more difficult to maintain attention leading to mind-wandering. Change detection tasks, in contrast, simply present an array of items, and after a brief delay, participants have to indicate if an item changed or not. Thus, there is no distracting activity in the task to disrupt attention. Furthermore, prior research indicates that mind-wandering tends to increase with slower paced tasks (e.g., Smallwood \& Schooler, 2006). In complex span tasks the trial sequence includes performing the distractor task for several seconds and being presented with a to-be-remembered item for approximately $1 \mathrm{~s}$. Thus, on some trials the overall trial sequence can take $20 \mathrm{~s}$ or more. On change detection tasks, however, the overall trial sequence typically only takes about $2 \mathrm{~s}$. Thus, it seems likely that mind-wandering would be expected in complex span tasks that include distracting activity and long trial sequences, but it is less clear whether mindwandering should occur during change detection tasks. Finally, in the literature there is typically a divide between those who use complex span tasks to measure WM and those that use change detection tasks to measure visual WM. Thus, overall, more work is needed to assess the extent to which capacity measures of WM (such as change detection tasks) are susceptible to mind-wandering as predicted by prior research.

The aim of the current study was to examine the extent to which individuals experience lapses of attention during capacity measures of visual WM, assess the extent to which lapses of attention are associated with poorer task performance, and finally assess the extent to which individual differences in lapses of attention are related to estimates of WM capacity. To examine this, participants performed fairly standard change detection tasks in which the number of items that were to-be-remember varied. Importantly, using thought-probe techniques, we queried participants on their current attentional state during these tasks to obtain a measure of lapses of attention.

\section{Experiment 1}

The purpose of Experiment 1 was to examine whether lapses of attention during capacity measures of WM are due to mindwandering. Prior research has suggested that participants 
mind-wander during many tasks requiring sustained attention and that lower ability individuals mind-wander more than high ability individuals (McVay \& Kane, 2012; Mrazek et al., 2012). To examine this notion, participants performed a standard change detection task and were probed randomly after some trials and asked to classify their immediately preceding thoughts. If lapses of attention influence capacity measures of WM, we should see that participants mind-wander during WM tasks, that mind-wandering is associated with lower task performance than when participants report being on-task, and that low capacity individuals should report mind-wandering more than high capacity individuals.

\section{Method}

\section{Participants}

Participants were 65 undergraduate students, recruited from the subject pool at the University of Oregon. Participants received course credit for their participation. Because some analyses would examine between-participant correlations we decided on a minimum sample size of 65 .

\section{Procedure}

Participants were tested individually and performed a change detection task with colored squares as the WM task. In this task participants were first presented with a black fixation cross in the middle of the screen on a gray background for 1,000 ms. Next, participants were presented with arrays of 4,6 , or 8 colored squares $\left(0.65^{\circ} \times 0.65^{\circ}\right)$ for $250 \mathrm{~ms}$. The arrays were arranged randomly on a neutral gray background, with each color randomly selected from one of seven easily discriminable colors (red, blue, violet, green, yellow, black, or white). The items in the arrays were separated by at least $2^{\circ}$ of visual angle measured from the centers of the square. The presentation of the arrays was followed by a delay period of $900 \mathrm{~ms}$, and finally the test array reappeared with one of the items circled. Participants responded as to whether or not the circled item had changed color. No performance feedback was given. Participants completed 162 total trials with 54 trials per set size. Set sizes were randomly presented. Half of the trials were change trials. Capacity $(\mathrm{K})$ was estimated using Cowan's (2001) formula for each set size and each individual. These values were then averaged to get an estimate of capacity.

During the task, participants were periodically presented with thought probes asking them to classify their immediately preceding thoughts. We used thought probes similar to those used by Unsworth and McMillan (2013), which asked participants to press one of six keys to indicate what they were thinking just prior to the appearance of the probe. Specifically, participants saw:

What were you just thinking about?

1. The current task

2. My performance on the current task

3. A memory from the past

4. Something in the future

5. Current state of being

6. Other

During the instructions participants were given specific instructions regarding the different categories. Similar to Unsworth and McMillan (2013; McVay \& Kane, 2012), Response 1 was classified as on-task, Response 2 was classified as task-related interference, and Responses 3 through 6 were classified as mind-wandering. Participants completed 162 trials with thought probes appearing after 30 trials (10 probes per set size).

\section{Results and discussion}

First, we examined mind-wandering rates during the task. Shown in Table 1 are the proportions of each thought-probe response. As can be seen, participants reported being on-task $54 \%$ of the time and mind-wandering $27 \%$ of the time. Furthermore, as shown in Table 2, accuracy was lower for trials where participants reported mind-wandering compared to when they reported being on task, $t(56)=3.46, p<.001$. Note this paired samples $t$ test is based only on those participants who reported being both on-task and mindwandering during the task. Mind-wandering rates for this subsample were comparable to the overall sample (i.e., 29\%), and on average participants contributed 8.98 $(S D=6.15)$ data points to the analysis. Thus, during a WM task participants reported frequent mind-wandering, and performance was worse on trials where participants reported mind-wandering compared to when they reported being on task.

Next, we examined whether individual differences in mind-wandering rates would predict capacity estimates of working memory. There was a significant negative correlation

Table 1 Proportions of each thought-probe response for Experiments 1-3

\begin{tabular}{llll}
\hline & On task & TRI & MW \\
\hline Experiment 1 & $.54(.28)$ & $.19(.21)$ & $.27(.21)$ \\
Experiment 2 & $.51(.29)$ & $.23(.20)$ & $.27(.22)$ \\
Experiment 3 & $.54(.30)$ & $.21(.19)$ & $.26(.24)$ \\
\hline
\end{tabular}

Note. $\mathrm{TRI}=$ task-related interference; $\mathrm{MW}=$ mind-wandering. Standard deviations are in parentheses 
Table 2 Proportion correct as a function of each thought-probe response for Experiments 1-3

\begin{tabular}{llll}
\hline & On task & TRI & MW \\
\hline Experiment 1 & $.88(.11)$ & $.84(.22)$ & $.74(.27)$ \\
Experiment 2 & $.91(.09)$ & $.85(.21)$ & $.84(.19)$ \\
Experiment 3 & $.91(.10)$ & $.85(.21)$ & $.84(.16)$
\end{tabular}

Note. TRI $=$ task-related interference; $\mathrm{MW}=$ mind-wandering. Standard deviations are in parentheses

between WM capacity and mind-wandering rates $(r=-.28$, $p=.026) .{ }^{1}$ Thus, low capacity individuals experienced more mind-wandering on a WM task than high capacity individuals.

Consistent with prior modeling research, the current results demonstrated that participants experience fluctuations of attention during capacity measures of WM, and these fluctuations are related to lower task performance. Furthermore, the results demonstrated that when participants experience lapses of attention performance is worse compared to when they report being on-task. Finally, low WM capacity individuals experience more mind-wandering and lapses of attention than high WM capacity individuals.

\section{Experiment 2}

The purpose of Experiment 2 was to examine whether lapses of attention and mind-wandering on small set sizes are partially responsible for less than perfect accuracy on those set sizes. Theoretically, as noted previously, most healthy young adults should be able to maintain a single item (one colored square) in WM over a brief delay. Yet, performance on small set sizes is not always perfect. Prior modeling research has suggested that less than perfect performance on small set sizes is due to participants experiencing periodic lapses of attention and that when a lapse parameter is added into WM models the data can be accounted for (R. D. Morey, 2011; Rouder et al., 2008). To see if this is the case, participants performed the same change detection task as the prior experiments but with set sizes of 1 , 4 , or 8 colored squares. If lapses of attention account for less than perfect performance on set sizes of 1 , then we should see that participants report mind-wandering on these set sizes and that performance is less than perfect when they report mindwandering but close to perfect when they report being on-task.

\footnotetext{
${ }^{1}$ In each experiment we also examined whether task-related interference (TRIs) correlated with estimates of capacity. In no case did TRIs correlate significantly with estimates of capacity (E1 $r=.12$, E2 $r=.18$, E3 $r=.03$ ). Additionally, TRIs did not correlate with filtering abilities in Experiment $3(r=-.14)$. Thus, not all attentional states are related to capacity estimates of WM.
}

\section{Method}

\section{Participants}

Participants were 72 undergraduate students recruited from the subject pool at the University of Oregon. Participants received course credit for their participation. Because some analyses would examine betweenparticipant correlations, we decided on a minimum sample size of 65 .

\section{Procedure}

Participants were tested individually and performed the same change detection task as in Experiment 1, except that the set sizes were 1,4 , or 8 colored squares.

\section{Results and discussion}

As shown in Table 1, participants again reported mindwandering on $27 \%$ of probe trials and performance on mindwandering trials was worse than on trials where they reported being on-task (see Table 2), $t(60)=2.60, p=.012$. Note this paired samples $t$ test is based only on those participants who reported being both on-task and mind-wandering during the task. Mind-wandering rates for this subsample were comparable to the overall sample (i.e., 31\%), and on average participants contributed $9.29(S D=6.22)$ data points to the analysis. Examining mind-wandering rates for each set size suggested that participants experienced mind-wandering on all set sizes (Set Size $1 M=.27, S D=.24$; Set Size $4 M=.26, S D=$ .25 ; Set Size $8 M=.27, S D=.24)$. Critically, examining Set Size 1 performance was worse on trials where participants reported mind-wandering $(M=.95, S D=$ .13) compared to trials where they reported being ontask $(M=.99, S D=.03), t(50)=2.44, p=.018$. Thus, participants report mind-wandering on small set sizes, and performance is less than perfect on small set sizes when participants report mind-wandering. Examining individual differences suggested a significant negative correlation between overall mind-wandering rates and estimates of capacity $(r=-.39, p<.001)$.

Consistent with Experiment 1, participants reported mind-wandering during a WM task, and performance was worse when participants reported mind-wandering compared to when they reported being on-task. Importantly, these results were found even for Set Size 1, suggesting that one reason participants demonstrate less than perfect performance on small set sizes is because they experience periodic lapses of attention and higher levels of inattention (R. D. Morey, 2011; Rouder et al., 2008; Unsworth \& Robison, 2015). 


\section{Experiment 3}

Thus far the current results suggest that attention control processes are needed during WM tasks in order to sustain attention and prevent lapses or fluctuations in attention across trials. Prior research has suggested that attention control processes are also needed during WM tasks when distractors are present (Vogel et al., 2005). In particular, Vogel et al. found that when participants were presented with red and blue rectangles and told only to remember the orientations of the red rectangles, the presence of the blue rectangles hurt performance, especially for low WM capacity individuals. Vogel et al. suggested that the ability to filter out distractors and prevent them from gaining access to WM is an important aspect of performance on WM tasks. In the current experiment we examined whether lapses of attention would influence performance on this task and, importantly, we examined whether the ability to prevent lapses of attention is related to the ability to filter distractors. Theoretically, both are forms of attention control, so it seems likely that they will be related and account for similar variance in capacity estimates of WM. At the same time, it is possible that these two attention control processes reflect distinct forms of attention control, both of which account for variability in capacity estimates. To examine this, participants performed a change detection task in which red and blue rectangles were presented, and participants were instructed to remember the orientations of the red rectangles and ignore the blue rectangles. Similar to the prior experiments, thought-probes were presented randomly after trials to assess the influence of mind-wandering and lapses of attention on performance.

\section{Method}

\section{Participants}

Participants were 109 undergraduate students, recruited from the subject pool at the University of Oregon. Participants received course credit for their participation. Because some analyses would examine between-participant correlations and examine whether two measures accounted for unique variance in capacity estimates, we decided on a minimum sample size of 100 .

\section{Procedure}

Participants were tested individually and performed a change detection task modeled after Vogel et al. (2005). In this task participants were first presented with a black fixation cross in the middle of the screen on a gray background for $1,000 \mathrm{~ms}$. Next participants were presented with arrays of two, four, or six red and/or blue rectangles for $250 \mathrm{~ms}$. Arrays consisted of two red rectangles alone, two red rectangles and two blue rectangles, four red rectangles alone, or four red rectangles and two blue rectangles. Participants were instructed to remember the orientations of the red rectangles and ignore the blue rectangles. Items were presented within a gray $19.1^{\circ} \times$ $14.3^{\circ}$ field. Items were separated from one another by at least $2^{\circ}$ and were all at least $2^{\circ}$ from central fixation. The presentation of the arrays was followed by a delay period of $900 \mathrm{~ms}$, and, finally, the test array reappeared with a white dot appearing in the middle of one of the items. Participants responded as to whether or not the orientation of the item with a white dot had changed. Participants completed 184 total trials, with 46 trials per condition. Trials were randomly presented. Half of the trials were change trials. Thought probes appeared after 40 trials ( 10 probes per condition). Capacity (K) was estimated using Cowan's (2001) formula for each set size and each individual. Filtering efficiency was estimated as the difference in accuracy for trials with no distractors versus trials with distractors. Larger values indicate a larger drop in performance when distractors are present.

\section{Results and discussion}

First we examined accuracy as a function of each target set size (two vs. four) and presence of distractors (zero vs. two). Figure 1 shows proportion correct for each condition. There was a main effect of target set size, $F(1,108)=274.84, M S E=.001, p<.001$, partial $\eta^{2}=.72$, suggesting that performance decreased with larger set sizes $(M=.93, S E=.01$ vs. $M=.83, S E=.01)$. There was also a main effect of distractor presence, $F(1,108)=$ 38.37, MSE $=.001, p<.01$, partial $\eta^{2}=.26$, suggesting that performance decreased when distractors were present $(M=.90$, $S E=.01$ vs. $M=.86, S E=.01)$. The interaction between these two was not significant, $p>.87$. Thus, consistent with prior research performance was reduced when distractors were presented and participants were required to filter them out.

Examining the thought-probe responses suggested that participants reported being on-task $54 \%$ of the time and mindwandering $26 \%$ of the time. Furthermore, as shown in Table 2, accuracy was lower for trials where participants reported mind-wandering compared to when they reported being on task, $t(82)=3.82, p<.001$. Note this paired samples $t$ test is based only on those participants who reported being both ontask and mind-wandering during the task. Mind-wandering rates for this subsample were comparable to the overall sample (i.e., 32\%), and on average participants contributed 13.08 $(S D=8.74)$ data points to the analysis. Similar to Experiment 2 we also examined performance on the smallest set size (two red alone) for trials where participants reported being on-task compared to mind-wandering. Similar to Experiment 2, performance was better on small set sizes when participants reported being on-task $(M=.97, S D=.10)$ compared to experiencing a lapse of attention $(M=.91, S D=.23), t(66)=$ 


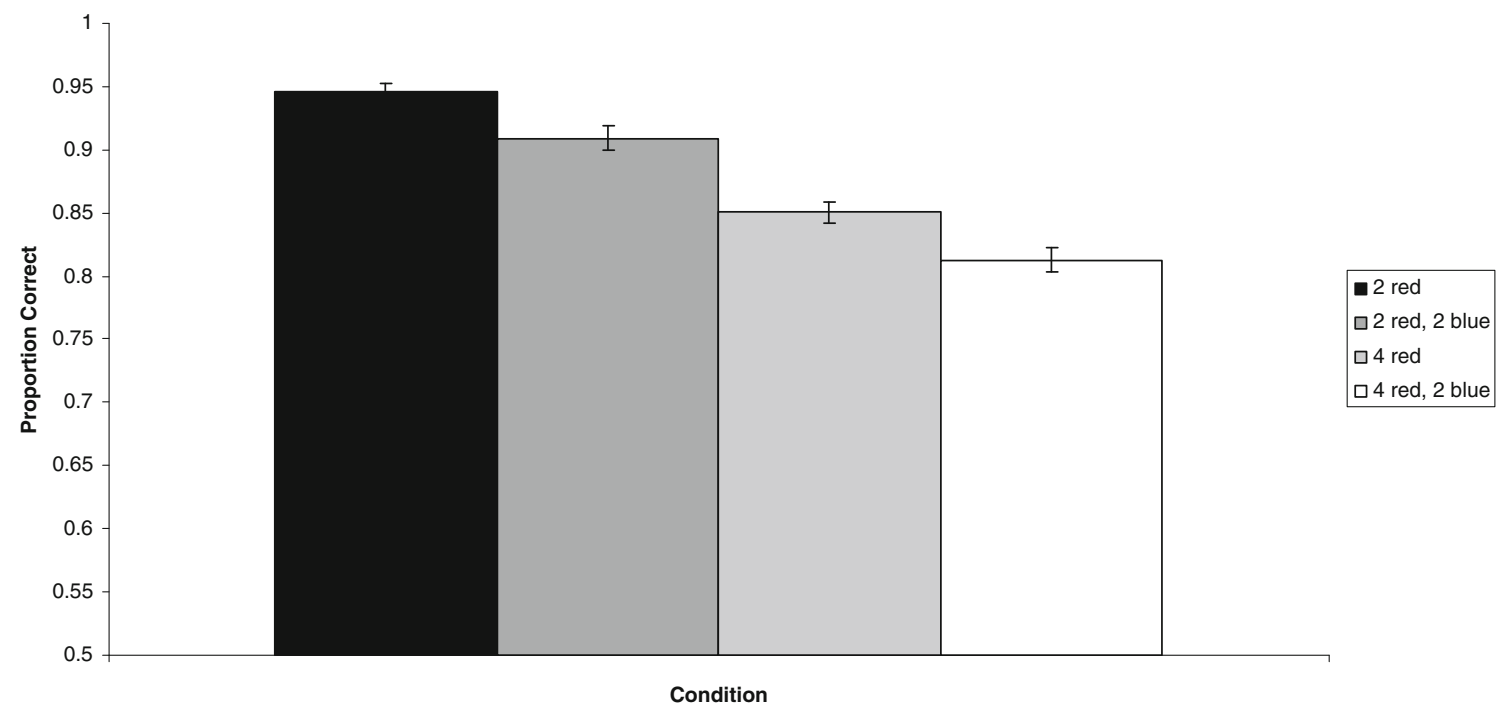

Fig. 1 Proportion correct as a function of each condition in Experiment 3. Error bars reflect 1 standard error of the mean

2.06, $p<.044$. Thus, consistent with the prior experiments participants experienced lapses of attention during a WM task and these lapses were associated with poorer performance than when participants reported being on-task and this occurred even at the smallest set size.

The final set of analyses examined the critical question of whether lapses of attention (based on mind-wandering rates) and filtering abilities would account for the same or different variance in capacity estimates (see Table 3 for descriptive statistics). Similar to the prior experiments, mind-wandering was correlated with capacity estimates $(r=-.31, p<.01)$ and, consistent with prior research, filtering was correlated with capacity estimates $(r=-.36, p<.001)$. Interestingly, mindwandering and filtering were not correlated $(r=.03, p>.72)$. To examine these relations in more detail, we submitted the mind-wandering and filtering values to a simultaneous regression predicting the estimates of capacity. As seen in Table 4, both mind-wandering and filtering accounted for unique variance in capacity. Collectively, mind-wandering and filtering accounted for $21 \%$ of the variance in capacity estimates. These results suggest that individual differences in capacity are driven, in part, by individual differences in the ability to filter out distractors during encoding and by the ability to prevent lapses of attention across trials.

Table 3 Descriptive statistics and reliability estimates for Experiment 3

\begin{tabular}{llllll}
\hline Measure & $M$ & $S D$ & Skew & Kurtosis & $\alpha$ \\
\hline MW & .26 & .24 & .97 & .55 & .94 \\
Filtering & .04 & .06 & 1.15 & 2.11 & .46 \\
K & 2.18 & .48 & -1.52 & 3.51 & .83
\end{tabular}

Note. $\mathrm{MW}=$ mind wandering; Filtering = filtering score; $\mathrm{K}=$ capacity estimate of working memory

\section{General discussion}

In three experiments, we examined whether lapses of attention occur during capacity measures of WM and whether these lapses are associated with poorer task performance. We found that when participants reported mind-wandering overall task performance was much lower than when participants rated their attentional focus as high or reported they were on-task. These effects occurred even on the smallest set sizes. Overall, these results suggest that lower levels of performance on some trials (including small set sizes) could be due to participants experiencing a lapse of attention, whereby the array is not fully encoded or actively maintained and participants must resort to guessing. Thus, the current results provide direct support for the lapse hypothesis suggested by prior modeling and pupillometry work (R. D. Morey, 2011; Rouder et al., 2008; Unsworth \& Robison, 2015). In line with the context-regulation hypothesis of mindwandering (Smallwood, 2013), the current results suggest that on attention-demanding tasks, like change detection tasks, mindwandering can have negative effects on performance, leading to lowered estimates of WM capacity. At the same time, some research suggests that on non-attention-demanding tasks, higher capacity individuals may actually mind-wander more, with little deficits to performance (see Smallwood \& Schooler, 2015, for a review). Thus, depending on the task context, there may be both costs and benefits to mind-wandering.

Table 4 Simultaneous regression predicting $\mathrm{K}$ for Experiment 3

\begin{tabular}{llllll}
\hline Variable & $B$ & $t$ & $s r^{2}$ & $R^{2}$ & $F$ \\
\hline MW & -.30 & $-3.51^{* *}$ & .09 & & \\
Filtering & -.35 & $-4.01^{* *}$ & .12 & .21 & $14.70^{* *}$ \\
\hline
\end{tabular}

$* * p<.01$ 
Examining individual differences, the current experiments demonstrated robust and modest relations between selfreports of attentional lapses and estimates of WM capacity. Across all three experiments, low WM capacity individuals reported less task focus and more mind-wandering than high WM capacity individuals. Additionally, examining the relation between sustaining attention and filtering abilities suggested that both of these attention control abilities accounted for unique variance in estimates of WM capacity. These results suggest that attention control abilities are needed to sustain attention on task across trials and prevent lapses of attention and to filter out distractors during encoding to ensure that only target items are maintained. Importantly, the current results suggest that these two abilities are somewhat distinct, with each accounting for important individual differences in WM capacity. These results are consistent with recent work by Forster and Lavie (2014), indicating that individual differences in mind-wandering were related to task-irrelevant distraction (see also Unsworth \& McMillan, 2014a), but individual differences in mind-wandering were not related to taskrelevant distraction in a response competition paradigm. Similarly, Barron, Riby, Greer, and Smallwood (2011) found that individual differences in mind-wandering were related with less processing of external stimuli, suggesting that while mind-wandering, attention is decoupled from the environment. Thus, the current results combined with prior research suggest that there may be separable components of attention control such that some control processes are needed to filter and block task-relevant distraction, and other control processes are needed to prevent lapses of attention to taskirrelevant distraction (which can be both internal, such as mind-wandering, and external). Future research is needed to better examine potential similarities and differences between these types of attention control and how they are used to ensure active maintenance of information in WM.

Furthermore, the results have important implications for estimating the capacity of WM with change detection (and potentially other) tasks. In particular, the current results suggest that it is possible that individuals who mind-wander frequently may have larger capacities than can be estimated based on their task performance. That is, it is possible that low WM capacity individuals can actually maintain the same number of items in WM as high capacity individuals on those trials where they are fully engaged in the task. However, because these same individuals experience more mindwandering throughout the task, their performance on some trials will be lower, leading to lower estimates of capacity (see also Adam et al., 2015). Additionally, it is possible that there are subgroups of individuals, some of which have attention control problems (but intact capacity) and others who have lower capacities (but intact attention control). Future research is needed to better examine the extent to which mind-wandering is leading to lower estimates of capacity and/or lowered capacity is leading to more mind-wandering. For now, the current results, along with prior research (Mrazek et al., 2012) suggest that mind-wandering occurs in a number of WM tasks, and susceptibility to mind-wandering in these tasks is related to individual differences in WM.

The current results also have important implications for theories of visual WM, which rely heavily on change detection tasks. In particular, although some models of visual WM suggest the need for a lapse parameter to account for the occasional error on small set sizes (R. D. Morey, 2011; Rouder et al., 2008), not all models have such a parameter. For example, resource models of visual WM suggest that memory resources are divided across items in the array, and as more items are presented in the array, fewer resources are allocated to each item (e.g., Bays \& Husain, 2008). Importantly, as they currently stand, these resource models do not account for mind-wandering and fluctuations in performance across trials. In order to do so, these models would need to assume that the continuous memory resource fluctuates across trials such that there are more resources on some trials than on others. Furthermore, it could be assumed in these models that the same resource that is allocated to items in WM can be allocated to self-generated thoughts (mind-wandering), and thus, when one engages in mind-wandering there are fewer resources available for the memory representations. Clearly, in principle, resource models could handle the current results (and others), but what is important is that these models either need to be augmented with an attention parameter that fluctuates across trials, or they need to better define exactly what the resources are and/or how these resources may be allocated to task-relevant and task-irrelevant representations.

The current results also have implications for slots models of visual WM, which suggest there are a discrete number of slots (usually four) in WM. Some prior slot models have suggested the need for a lapse parameter, but these models typically assume that lapses occur in an all-or-none fashion such that you are either fully engaged in the task or you have experienced a lapse of attention and must resort to guessing (R. D. Morey, 2011; Rouder et al., 2008). Although this seems plausible, it also seems possible that lapses occur in a more graded fashion, such that participants are only partially engaged in the task or only partially mind-wandering such that enough attention is devoted to the task so that performance isn't horrible (e.g., Adam et al., 2015). Indeed, in some of our prior research we found that on difficult reasoning tasks, and the Stroop task, participants indicated only being $70 \%$ focused on the task at any given time (Unsworth \& McMillan, 2014b. 2014c). Thus, although some prior models of visual WM have indicated a need for a lapses parameter to account for the occasional error (especially on smaller set sizes), it seems that more work is needed to more fully account for the how fluctuations in attention and mind-wandering influence performance on visual change detection tasks and what this means for theories of visual WM. 
Finally, the current results have implications for the notion that maintaining items in WM results in storage in long-term memory (e.g., Atkinson \& Shiffrin, 1968). In particular, the current results suggest that if participants mind-wander while encoding and maintaining items in WM, then there should be negative downstream consequences for storage in long-term memory, with these representations not being stored very well or at all. Indeed, prior research has shown that mindwandering during memory encoding is associated with poorer subsequent recall than when participants report being on task (Smallwood, Baracaia, Lowe, \& Obonsawin, 2003). Thus, mind-wandering during WM tasks not only can lead to reductions in WM performance but also can lead to poorer subsequent recall from long-term memory. Collectively, the present results suggest the overall importance of examining how lapses of attention during WM tasks can influence not only performance on those tasks, but influence relations between WM and other cognitive systems.

A potential alternative explanation for the current results is that perhaps poor task performance led to increased reports of mind-wandering. That is, perhaps participants knew that they made an error on a particular trial, and when the probe appeared they justified their performance by indicating that they had been mind-wandering. Although possible, we think this explanation is unlikely to account for the current results for a number of reasons. First, as noted in the method, performance feedback was not given during the task. Thus, participants did not always know when they had made an error, especially on larger set sizes where guessing is likely. Second, the notion that poor performance leads to higher rates of mind-wandering would predict that mind-wandering rates should be highest in conditions with the worst performance. However, as reported in Experiment 2, there were roughly equal rates of mindwandering in Set Sizes 1, 4, and 8. Third, even though trials where mind-wandering was reported were less accurate than trials of on-task reports, the mind-wandering trials were still associated with relatively high accuracy. Indeed, in Experiment 2, participants reported mind-wandering on 27\% of Set Size 1 trials, yet accuracy was still above 90\%. Thus, participants were reporting mind-wandering more often after correct than after incorrect trials. This may suggest that participants' reports are not accurate, but it should be noted that our prior pupillometry work suggests that participants are not allocating much attention on small set sizes. Thus, it is possible for participants to not be completely focused on the task and still perform well. However, on some small proportion of trials (especially for low capacity individuals), this lack of focus will lead to errors. Finally, it should be noted that nowhere in the probe responses is mind-wandering explicitly mentioned. Rather the probes ask whether participants were thinking about the current task, their performance on the current task, a memory from the past, something in the future, or their current state of being. If participants were basing their responses solely on their performance, one would expect that mind-wandering rates would be associated with higher reports of task performance (task-related interference). However, as noted in Footnote 1, task-related interference was not related to capacity estimates in any of the experiments. Thus, overall it does not seem that the current results are simply due to participants reporting mind-wandering after poor task performance.

\section{Conclusions}

Collectively, the current results suggest that the ability to focus and sustain attention on task and prevent lapses of attention is an important contributor to performance on capacity measures of WM, such as visual change detection tasks. Those individuals who can consistently sustain attention on-task and prevent lapses of attention will be better able to encode and maintain items in WM, leading to higher estimates of capacity. Individuals susceptible to lapses of attention will fail to properly encode or maintain items in WM, leading to overall lower estimates of capacity. By measuring subjective attentional state across trials, the current techniques provide a promising means for examining lapses of attention during WM capacity tasks and further elucidating the role of attention control in WM operations.

\section{References}

Adam, K. C. S., Mance, I., Fukuda, K., \& Vogel, E.K. (2015). The contributions of attentional lapses to individual differences in working memory capacity. Journal of Cognitive Neuroscience, 27, 16011616. doi:10.1162/jocn_a_00811.

Atkinson, R. C., \& Shiffrin, R. M. (1968). Human memory: A proposed system and its control processes. In K. W. Spence (Ed.), The psychology of learning and motivation (Vol. 2, pp. 89-195). New York: Academic Press.

Barron, E., Riby, L. M., Greer, J., \& Smallwood, J. (2011). Absorbed in thought: The effect of mind wandering on the processing of relevant and irrelevant events. Psychological Science, 22, 596-601.

Bays, P. M., \& Husain, M. (2008). Dynamic shifts of limited working memory resources in human vision. Science, $321,851$.

Cowan, N. (2001). The magical number 4 in short-term memory: A reconsideration of mental storage capacity. Behavioral and Brain Sciences, 24, 97-185.

Cowan, N., Elliott, E. M., Saults, J. S., Morey, C. C., Mattox, S., Hismjatullina, A., \& Conway, A. R. A. (2005). On the capacity of attention: Its estimation and its role in working memory and cognitive aptitudes. Cognitive Psychology, 51, 42-100.

Cowan, N., Fristoe, N. M., Elliot, E. M., Brunner, R. P., \& Saults, J. S. (2006). Scope of attention, control of attention, and intelligence in children and adults. Memory \& Cognition, 34, 1754-1768.

Donkin, C., Nosofsky, R. M., Gold, J. M., \& Shiffrin, R. M. (2013). Discrete-slots models of visual working-memory response times. Psychological Review, 120, 873-902. 
Engle, R. W., \& Kane, M. J. (2004). Executive attention, working memory capacity, and a two-factor theory of cognitive control. In B. Ross (Ed.), The psychology of learning and motivation (Vol. 44, pp. 145199). New York: Elsevier.

Forster, S., \& Lavie, N. (2014). Distracted by your mind? Individual differences in distractability predict mind wandering. Journal of Experimental Psychology: Learning, Memory, \& Cognition, 40, 251-260.

Larson, G. E., \& Alderton, D. L. (1990). Reaction time variability and intelligence: A "worst performance" analysis of individual differences. Intelligence, 14, 309-325.

McVay, J. C., \& Kane, M. J. (2009). Conducting the train of thought: Working memory capacity, goal neglect, and mind wandering in an executive-control task. Journal of Experimental Psychology: Learning, Memory, and Cognition, 35, 196-204.

McVay, J. C., \& Kane, M. J. (2012). Why does working memory capacity predict variation in reading comprehension? On the influence of mind wandering and executive attention. Journal of Experimental Psychology: General, 141, 302-320.

Morey, R. D. (2011). Hierarchical Bayesian models of working memory capacity. Journal of Mathematical Psychology, 55, 8-24.

Morey, C. C., Cowan, N., Morey, R. D., \& Rouder, J. N. (2011). Flexible attention allocation to visual and auditory working memory tasks: Manipulating reward induces a trade-off. Attention Perception, \& Psychophsyics, 73, 458-472.

Mrazek, M. D., Smallwood, J., Franklin, M. S., Chin, J. M., Baird, B., \& Schooler, J. W. (2012). The role of mind-wandering in measurements of general aptitude. Journal of Experimental Psychology: General, 141, 788-798.

Rouder, J., Morey, R. D., Cowan, N., Zwilling, C. E., Morey, C. C., \& Pratte, M. S. (2008). An assessment of fixed-capacity models of visual working memory. Proceedings of the National Academy of Sciences of the United States of America, 105, 5976-5979.

Shipstead, Z., Lindsey, R. B., Marshall, R. L., \& Engle, R. W. (2014). The contributions of maintenance, retrieval and attention control to working memory capacity. Journal of Memory and Language, 72, $116-141$

Smallwood, J. (2013). Distinguishing how from why the mind wanders: A process-occurrence framework for self-generated mental activity. Psychological Bulletin, 139, 519-535.

Smallwood, J., \& Schooler, J. W. (2006). The restless mind. Psychological Bulletin, 132, 946-958.
Smallwood, J., \& Schooler, J. W. (2015). The science of mindwandering: Empirically navigating the stream of consciousness. Annual Review of Psychology, 66, 487-518.

Smallwood, J., Baracaia, S. F., Lowe, M., \& Obonsawin, M. C. (2003). Task-unrelated-thought whilst encoding information. Consciousness and Cognition, 12, 452-484.

Unsworth, N., \& Engle, R. W. (2007). The nature of individual differences in working memory capacity: Active maintenance in primary memory and controlled search from secondary memory. Psychological Review, 114, 104-132.

Unsworth, N., \& McMillan, B. D. (2013). Mind wandering and reading comprehension: Examining the roles of working memory capacity, interest, motivation, and topic experience. Journal of Experimental Psychology: Learning, Memory, \& Cognition, 39, 832-842.

Unsworth, N., \& McMillan, B. D. (2014a). Similarities and differences between mind-wandering and external distraction: A latent variable analysis of lapses of attention and their relation to cognitive abilities. Acta Psychologica, 150, 14-25.

Unsworth, N., \& McMillan, B. D. (2014b). Trial-to-trial fluctuations in attentional state and their relation to intelligence. Journal of Experimental Psychology: Learning, Memory, \& Cognition, 40, 882-891.

Unsworth, N., \& McMillan, B. D. (2014c). Fluctuations in pre-trial attentional state and their influence on goal neglect. Consciousness and Cognition, 26, 90-96.

Unsworth, N., \& Robison, M. K. (2015). Individual differences in the allocation of attention to items in working memory: Evidence from pupillometry. Psychonomic Bulletin \& Review, 22, 757-765.

Unsworth, N., Redick, T. S., Lakey, C. E., \& Young, D. L. (2010). Lapses in sustained attention and their relation to executive and fluid abilities: An individual differences investigation. Intelligence, 38, 111-122.

Unsworth, N., Fukuda, K., Awh, E., \& Vogel, E. K. (2014). Working memory and fluid intelligence: Capacity, attention control, and secondary memory retrieval. Cognitive Psychology, 71, 1-26.

Vogel, E. K., McCollough, A. W., \& Machizawa, M. G. (2005). Neural measures reveal individual differences in controlling access to visual working memory. Nature, 438, 500-503.

Weissman, D. H., Roberts, K. C., Visscher, K. M., \& Woldorff, M. G. (2006). The neural bases of momentary lapses of attention. Nature Neuroscience, 9, 971-978. 